\title{
"Surprisingly, there is an actual physical application..." Student understanding in Math Methods
}

\author{
Michael E. Loverude*† and Sissi L. Li† \\ *Department of Physics MH-611, California State University Fullerton, Fullerton, CA, 92834 \\ † Catalyst Center, MH-531, California State University Fullerton, Fullerton, CA, 92834
}

\begin{abstract}
Among the canonical physics core courses taken by most undergraduate majors is a course in mathematical methods. Physics education research has begun to explore upper division physics courses, as well as the use of mathematics throughout the physics curriculum. The math methods course is an especially opportune environment to study the development of conceptual understanding of key ideas in mathematics and physics as well as the development of broadly applicable skills and the sociocultural norms of physics. In this poster we will explore some of what happened in a particular math methods course, with attention to the development of student content understanding as well as the development of community norms.
\end{abstract}

Keywords: math methods, upper division, physics education research.

PACS: Replace this text with PACS numbers; choose from this list: http://www.aip.org/pacs/index.html

\section{INTRODUCTION}

Physics education research (PER) has begun to make significant inroads into courses beyond the introductory level, with researchers studying student learning in most of the core courses for physics majors. A course in mathematical methods is required in many physics degree programs but this course has received relatively little attention in PER. A group at the University of Colorado has done work in combined math methods / classical mechanics courses that has some overlap [1, 2]. Numerous previous studies have investigated student use of math in physics, both at the introductory level [3-9] and in upper-division courses [2, 10-12].

\section{Context for Research}

This work has taken place in the context of a course titled Mathematical Methods of Physics taught at California State University Fullerton (CSUF). CSUF is a large public comprehensive university serving a diverse student population. The Math Methods course, Physics 300, is required for physics majors and is a prerequisite for upper-division theory courses; for most students it is one of the first upper-division courses taken. The course uses the text by Boas [1] and covers a fairly standard list of topics. It meets for two 75-minute blocks per week. The course has as prerequisites three semesters of calculus, and most students have completed at least two semesters of introductory physics. The author has taught the course four times, with enrollments between 12 and 19. A significant portion of class time is spent on interactive tutorial exercises but those are not the focus of this paper [13].

\section{Research Methods}

In the project as we have sought to document student understanding of the target ideas using standard methods of PER. In this paper we describe initial work in this course. The data source for conceptual understanding is student responses from written problems posed on ungraded quizzes given in class. We also illustrate data drawn from reflective essays collected via the online course management system. In all cases, student responses were collected, analyzed, and categorized by the researcher. 


\section{CONCEPTUAL UNDERSTANDING}

One key thread of this project has been to document student conceptual understanding of key elements of course content, both mathematical and physical in nature. We have used written problems on graded and ungraded quizzes and course examinations; these questions have been qualitative or semiqualitative in addition to more traditional quantitative problems. At this initial stage of the research, a guiding research question has been: to what extent do students entering the Math Methods course understand prerequisite math and physics content?

For the purpose of this paper, we highlight two sample tasks that illuminate key aspects of the math methods course and the relationship between physics and mathematics that this course brings to the fore. In both cases the data reflects student understanding before any instructional treatment, so student responses reflect initial understanding and prior coursework.

\section{Math that slips through the cracks: complex exponentials and oscillations}

Some math skills considered essential for physics seem to 'slip through the cracks' of students' prior training. When the lead author was first assigned to the course, he contacted instructors of the core theory courses for which Math Methods is a prerequisite. One instructor noted that students seemed to have difficulty with complex exponentials; Subsequent investigation revealed some previous study of this question [14].

For this study, students were given an ungraded quiz in which they were asked to sketch on a blank graph the real part of the function $f(t)=A e^{i \omega t}$ as a function of time and identify any relevant points. The expectation was that students would sketch a cosine function with amplitude A (i.e., value A at $t=0)$. This problem was posed in four sections of the course $(N=49)$, after reading and preliminary lecture on the topic but before a tutorial exercise on complex numbers. Students informally professed familiarity with Euler's equation $\left(e^{i x}=\cos x+i \sin x\right)$ from previous courses.

Student responses were categorized based on the following features: oscillatory or not, value at $\mathrm{t}=0$, constant or varying amplitude. Ten percent of responses could not be classified with this scheme and required an additional category; these responses included an arrow (possibly to represent a phasor in the complex plane) rather than a sketch of $f(t)$.

Of the student responses, 30\% were categorized as correct: a function that is oscillatory with a nonzero value at $t=0$. Another $10 \%$ were categorized as correct with incorrect phase (zero value at $t=0$ ). Over $40 \%$ of responses were categorized as not including oscillation; most of these sketches were consistent with exponential growth.

This raises questions about whether these students will be able to apply this mathematical formalism to physical contexts without further assistance. That finding resonates with previous research; Sadaghiani reported that $20 \%$ of students on one problem chose answers indicating that $e^{k x}$ and $e^{i k x}$ were solutions to the same differential equation, and that a similar number gave an answer to a potential well problem indicating oscillations when an answer for exponential growth/decay would be appropriate [14].

\section{Physicist skills in math problems: series expansions}

A second example illustrates a gap between physics and math usage of mathematical ideas. In this case, the problem (see Fig 1) requires that students use a series expansion to determine an approximate result given a mathematical expression. The question was posed as part of an ungraded quiz on the first day of two sections of the class $(N=28)$, before any instruction. 
Imagine that you are solving an E\&M problem and you derive the expression $E=\frac{+k Q}{\left(y-\frac{d}{2}\right)^{2}}+\frac{-k Q}{\left(y+\frac{d}{2}\right)^{2}}$ for the electric field at a certain location, with $\mathrm{y}>>\mathrm{d}$.

Use the binomial expansion to construct the first three terms of a series for [the first] term given that $\mathrm{y}>>\mathrm{d}$. Explicitly identify what $x$ and $p$ you are using. What simplification is allowed by the fact that $\mathrm{y}>>\mathrm{d}$ ?

FIGURE 1. A problem posed to students on the first day of the Math Methods course. An algebraic version of the binomial expansion was given to students.

Very few students produced complete and correct responses to the task. A third of the students left the problem completely blank, despite having ample time to respond. Analysis of the remaining responses suggest that students were unable to transform the expression into a form suitable for the expansion, i.e., $(1+x)^{p}$ with $x$ dimensionless. (In this case the series will only converge and be truncated if $|x|<<1$.) A key task in performing this expansion is to create a dimensionless quantity for a power series expansion (in this case, $\mathrm{d} / 2 \mathrm{y}$ ). We examined all student responses but only $10 \%$ of responses included such a dimensionless quantity.

Student responses to this task further suggest the importance of the interplay between mathematical calculation and more qualitative physical reasoning. The largest group of responses stated incorrectly that the electric field would be zero. The explanations and written work indicate two paths to this incorrect answer.

One group (20\% of students) responded that the electric field is zero because $y$ is large. Answers in this category tended to refer to the weakening of the field with distance exclusively and not an explicit calculation. This might reflect a 'dying away' resource and/or a more reasoned Coulomb's law argument. Another group (30\%) made an algebraic calculation to reach this conclusion, stating explicitly that $y>>d$ allows one to set $d=0$ or set $y-d / 2=y$. (The expression for $E$ then reduces to zero.)

While both categories of statement might be appropriate in certain circumstances, in this case they essentially eliminate all the interesting physics of the dipole configuration, in which the slight extra distance to one charge has important physical consequences. One might read these responses as two sides of the same underlying incorrect idea, and it is not clear whether the students in the second group force the math to support an intuition that $E=0$ or simply perform the calculations and reach that result.

\section{NORMS AND EXPECTATIONS}

While we have focused extensively on conceptual understanding, a significant goal of this project has been to document other aspects of the course. As noted, this course is one of the first entries to the world of upperdivision courses for our physics majors. As such, the course plays an important role in establishing norms and forming expectations. We believe that the process of enculturation plays a significant role in the development of physics majors, so we have examined this process in this course among others. We have also examined student expectations in the course, particularly as it relates to the relationship between math and physics, and what the purpose of math is in physics courses. For this paper, we will briefly highlight one aspect of this latter question.

We present selected student responses from a reflective essay assigned to students at the conclusion of the course. Students were asked to give advice to students taking the course in the future, and comment on what they saw as the important ideas of the course. 
The responses to this prompt included some process oriented statements ('read the book') but several reflect the underlying tension between math and physics:

[do] not treat the course as just a math course. ... be ready to use a lot of improvisation; I beleive [sic] that a major component of this course required that I try to correspond a mathematical method to a certain physics concept on the spot.

A second student also articulated a difference between math and physics, and suggests that his understanding of the purpose of the course evolved through the semester.

I feel that the core idea of this class was to bridge the gap between math and physics. This realization occured to me during the last two weeks when we covered eigenvalues. Surprisingly, there is an actual physical application.

A third student was in the somewhat unusual situation of taking the course at the same time as he took junior level electricity and magnetism, for which math methods is normally a prerequisite. This student wrote:

I would emphasize that it is beneficial to have a clear understanding of these mathematical tools ... so that when problems appear later that require them, one can focus on the physics revealed by them rather than just trying to figure out how the math works.

Each of these students make a point of distinguishing between 'math' and 'physics.' In particular the language used is revealing. Students describe a 'gap' and write about math 'method' and 'tools.' In contrast physics is described in terms of a 'concept' and an 'application.' In future work we will examine this relationship further.

\section{CONCLUSION AND IMPLICATIONS}

The results presented here are very preliminary, but a few tentative conclusions may be drawn. Students entering this course often still struggle with mathematical and physical reasoning that is expected by their instructors. The series expansion and complex exponential illustrated above are in principle covered in prerequisite math courses, but students have difficulty applying what they might have learned. Instruction in this course needs to consider the relationship between math and physics and help students make this connection. For example, the series expansion above is an authentic physics example. A simpler expression would simplify student calculations (and assessment), but students need to use this technique 'in the wild,' with expressions that include constants and other parameters and thus have dimensionality.

\section{REFERENCES}

1. M. Boas, Mathematical Methods in the Physical Sciences, Wiley (2006).

2. See arXiv:1207.0987v2 [physics.ed-ph] SEI: Science Education Initiaitive at University of Colorado Boulder, http://www.colorado.edu/sei/departments/physics.htm

3. B. L. Sherin, "How students understand physics equations," Cogn. and Instr. 19, 479-541 (2001).

4. E. F. Redish, "Problem solving and the use of math in physics courses," Proceedings of World View on Physics Education (2005).

5. D. E. Meltzer, "The relationship between mathematics preparation and conceptual learning gains in physics: A possible "hidden variable" in diagnostic pretest scores," American Journal of Physics, 70(12), 1259-1268 (2002).

6. D. C. Meredith and K. A. Marrongelle, "How students use mathematical resources in an electrostatics context," American Journal of Physics 76 (6) pp. 570 (2008).

7. D. H. Nguyen, \& N. S. Rebello, "Students' understanding and application of the area under the curve concept in physics problems," Phys. Rev. Spec. Topics: PER, 7(1), 1-17, (2011a).

8. D. H. Nguyen, \& N. S. Rebello, "Students' difficulties with integration in electricity," Phys. Rev. Spec. Topics: PER, 7(1), 010113, 1-11 (2011b).

9. R. E. Pepper, S. V. Chasteen, S. J. Pollock, and K. K. Perkins, "Observations on student difficulties with mathematics in upper-division electricity and magnetism," Phys. Rev. Spec. Topics: PER, 8 (1), 010111 1-15 (2012).

10. E. C. Sayre and M. C. Wittmann, Plasticity of intermediate mechanics students' coordinate system choice, Phys. Rev. Spec. Topics: PER, 4, 020105 (2008).

11. T.I. Smith, J.R. Thompson and D.B. Mountcastle, "Student Understanding of Taylor Series Expansions in Statistical Mechanics," Phys. Rev. Spec. Topics: PER, 9, 020110 (2013).

13. M.E. Loverude, "Assessment to match research-based instruction in upper-division courses," AIP Conference Proceedings 1413 (2011).

14. H. R. Sadaghiani, "Conceptual and mathematical barriers to students learning quantum mechanics," Ph.D. Dissertation, the Ohio State University (2005). 